\title{
Why Is the Moon Gradually Moving away from the Earth
}

\author{
Cuixiang Zhong \\ Department of Physics, Jiangxi Normal University, Nanchang, China
}

\begin{abstract}
Finding the cause of the Moon leaving the Earth is the first key to open the door of cosmic galaxies. Although some people believe the cause of the Moon leaving the Earth to be the tidal bulges on the Earth exerting a gravitational pull on the Moon, which tries to "speed up" the Moon, yet careful research shows this view is doubtful. Thus the author has studied the real causes for the Moon moving away from the Earth again, and found that the migration of the Moon away from the Earth is mainly due to the impacts of prograde planetsimals and the contraction of the growing Earth. Other satellites leaving their parent planets for the same reason.
\end{abstract}

Keywords: Moon; Earth; satellite; planet; orbit-variation.

\section{Introduction}

On July 20, 1969, USA “Appollo No. 11" spacecraft carrying three astronauts successfully landed on the moon. During the astronauts' landing on the Moon, they set up a laser reflecting device on the surface of the moon, making scientists be able to emit laser from the earth to the device and use the laser round-trip time to measure the distance between the Earth and the moon. The later measurement results show that the moon is moving away from the earth about $3.8 \mathrm{~cm}$ per year [1].

Why the Moon is moving away from the Earth? Some people believe this is caused by the Earth's tides raised by the Moon: because the tidal bulges on the Earth exert a gravitational pull on the Moon. The Earth rotates faster (once every 24 hours) than the Moon moves in its orbit (once every 27.3 days), thus the bulge tries to "speed up" the Moon, making the Moon move away from the Earth [2]. In fact, they have distorted Newton's theory of tides. Although Newton had proved that the Moon's gravitational pull can raise the tides on the Earth, he didn't mention that the tidal bulges on the Earth can speed up Moon's revolution. Since the mass of a tidal bulge is much

Corresponding author: Cuixiang Zhong, Dr., research fields: astrophysics and software applications. E-mail: cuixiang_zhong@163.com. less than the mass of the Moon, it has almost no gravitational pull on the Moon. Even if a raised wave ahead has a little gravitational pull on the Moon, a raised wave behind has also a little gravitational pull on the Moon, which offsets the gravitational pull of the wave ahead.

In addition, Mars has two natural moons, Phobos and Deimos, which orbit close to the planet, but Mars has little water. So we can conclude that it isn't Mars' tides that lead the orbit-variation of these two moons. But what is the real cause makes the moons moving away from their parent planet? In order to answer these questions, we should analyze various factors that cause moon's orbit-variation.

\section{The Real Causes of the Moon Leaving the Earth}

Through analyzing every possible force exerting on the Moon, we have discovered several reasons for the Moon moving away from the Earth:

(1) one reason is that prograde planetsimals' impacts on the Moon could speed up the Moon's revolution around the Earth, increasing the centrifugal force of the Moon, therefore making the moon move away from the Earth.

Many scientific studies have shown that, about 4.5 billion years ago, at the beginning of the formation of 
the Earth, frequent volcanic eruptions began to occur on the Earth, because then the Earth was much closer to the Sun and moved much more quickly around the Sun than now, making immense heat be accumulated inside the Earth, thus causing frequent and violent volcanic eruptions. During some violent volcanic eruptions or celestial body's impacts on the Earth, obviously the probability of volcanic eruptions is much larger than that of celestial body's impacts, some volcanic ash or rock debris could have achieved a velocity no less than the first cosmic velocity to enter some orbits around the Earth, forming layers of nebular around the Earth. Especially, when the direction of a volcanic eruption coincide with the direction of the Earth's rotation, the erupted debris could easily achieve a velocity no less than the first cosmic velocity; when the direction of a volcanic eruption contradicts with the direction of the Earth's rotation, the erupted debris have more difficulty achieving such a velocity. So there were more prograde debris than retrograde debris in the same orbit around the Earth. Hence, the prograde planetsimals could merge more prograde particles and bump less into retrograde particles, therefore easily grow up to be a Moon. That is why the Moon is a prograde satellite [3].

The orbit of the young Moon was much closer to the Earth than it is today. There were a lot of Earth ejecta in the orbit around the Earth, such as volcanic ash, water vapor, and aerosol, which could stay in the stratosphere for a very long time. Hence, the Moon, like a snowball, has unceasingly merged these ejecta to become larger and larger, and farther and farther away from the Earth. This can be proved as follows: When the Moon moved around the Earth normally, the centrifugal force produced by the Moon's rotation around the Earth and the Earth's gravitation pull on the Moon had the same size but opposite directions, as is shown in Fig. 1.

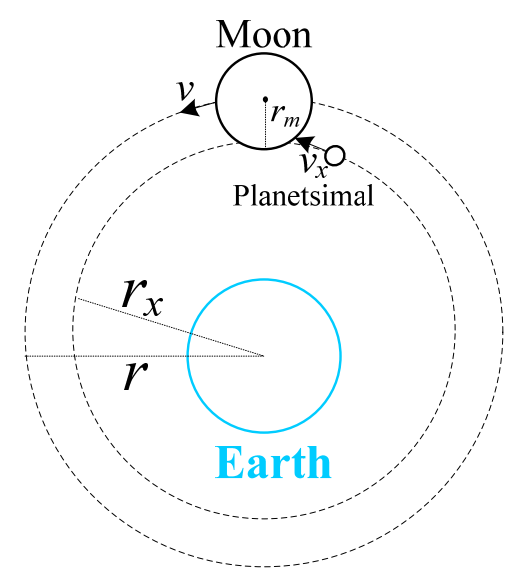

Fig. 1 The orbits of the Moon and planetsimals.

Let $\mathrm{M}$ be the mass of the Earth, $m_{1}$ be the mass of the Moon, $r_{\mathrm{m}}$ be the radius of the Moon, $r$ be the centroid distance between the Earth and the Moon, $v$ be the tangential velocity of the Moon around the Earth, then:

$$
\frac{G m_{1} M}{\mathrm{r}^{2}}=\frac{m_{1} v^{2}}{r} \rightarrow v=\sqrt{\frac{G M}{r}}
$$

Near the orbit of the Moon, there were also many smaller prograde planetsimals moving around the Earth's centre in circular orbits of radius $r_{x}\left(r-r_{m}<r_{x}<r\right)$ with velocity $v_{\mathrm{x}}$. Since

$$
v_{x}=\sqrt{\frac{G M}{r_{x}}}>\sqrt{\frac{G M}{r}}
$$

which implies $v_{\mathrm{x}}>v$, these smaller planetsimals would finally catch the Moon. When a smaller planetsimal approached the Moon, the Moon's gravitation force would accelerate the motion of the planetsimal, making the planetsimal's velocity become much larger than $v_{\mathrm{x}}$. Let $m_{2}$ be the mass of the planetsimal, $v_{\mathrm{y}}$ be its velocity when it impacted the Moon, then the centrifugal force of the Moon merged with the planetsimal was:

$$
m_{1} \cdot \frac{v^{2}}{r}+m_{2} \cdot \frac{v_{y}{ }^{2}}{r}>\left(m_{1}+m_{2}\right) \cdot \frac{v^{2}}{r}=G\left(m_{1}+m_{2}\right) \cdot \frac{\mathrm{M}}{r^{2}}
$$

That is, the centrifugal force produced by the Moon's rotation around the Earth was larger than the 
Earth's gravitation pull on the Moon, therefore the Moon's center of gravity has a trend moving away from the Earth.

Especially, if a planetsimal or asteroid was large enough, it would impact the Moon fiercely, making the Moon's velocity increase to a larger value $v_{2}$, then:

$$
\left(m_{1}+m_{2}\right) \cdot \frac{v_{2}^{2}}{r}>\left(m_{1}+m_{2}\right) \cdot \frac{v^{2}}{r}=G\left(m_{1}+m_{2}\right) \cdot \frac{\mathrm{M}}{r^{2}}
$$

therefore the Moon's center of gravity moved a reasonable distance from the Earth.

(2) Another reason is the increase in the Earth's rotation speed.

During the normal revolution of the Earth around the Sun, the atmospheric pressure on the trailing hemisphere of the Earth is higher than the other hemisphere, effectively increasing its revolution speed, thus making the Earth gradually move away from the parent-star along a spiral line [4]. In addition, with the parent star's shrinking from time to time and thus the speeding up of its rotation, the revolution speed of the Earth will also increase correspondingly and thus the Earth will move away from its parent star gradually. As the Earth gradually move away from their parent star, the gravitational attractions of the parent star to the Earth also gradually become smaller, consequently the Earth' self-rotation will gradually accelerate. The observation result of American National Institute of Standard Technology in 1999 also shows that the Earth's rotation is speeding up, which makes the moon' s revolution faster, eventually causing the Moon to move away from their planet.

(3) The third reason is that the Earth contracts from time to time, which is the main reason for the Moon moving away from the Earth.

With the growth of the mass of a planet, the temperature of planetary core strata gradually rises under increasing pressure, making the core strata melt into magma. When a large quantity of magma encounters external infiltrated water, it will be cooled and solidified, contracting the planet's volume but conserving the angular momentum of the planet, so a decrease in the rotational inertial results in an increase in the rotation speed of the planet, and consequently also an increase in the revolution speed of the Moon, making the Moon move away from the planet. For example, 2011 Tohoku earthquake has shifted Earth's figure axis by about 25 centimeters and accelerated Earth's spin [5].

\section{The Real Cause of Mars' Moons Leaving Their Parent Planet}

Mars' moons leave their parent planet for the same reason.

At one-tenth Earth's mass, Mars is too small to hold onto an appreciable atmosphere. Liquid water cannot exist on the surface of Mars due to low atmospheric pressure, which is less than $1 \%$ of the Earth's. So Mars' moons moving away from their parent planet is not caused by the Mars' tides raised by the moons. But the two polar ice caps appear to be made largely of water [6]. The volume of water ice in the south polar ice cap, if melted, would be sufficient to cover the entire planetary surface to a depth of 11 meters (36 ft) [7]. In November 2016, NASA reported finding a large amount of underground ice in the Utopia Planitia region of Mars. The volume of water detected has been estimated to be equivalent to the volume of water in Lake Superior on Earth. When Mars' magma encounters infiltrated water, it will be cooled and solidified, contracting the planet's volume but conserving the angular momentum of the planet, so a decrease in the rotational inertial results in an increase in the rotation speed of the planet, and consequently also an increase in the revolution speed of the moons, making the moons move away from their parent planet.

\section{Conclusion}

To sum up, the main factors causing the Moon move away from the Earth include the impacts of prograde planetsimals and the drag of the gravitational force of the Earth whose rotation is speeding up 
gradually. Other satellites leave their parent planet for the same reason. Having found such orbit-variation mechanisms of the Moon, we could reveal the formation and evolution of the Moon, the Solar System and other galaxies in the Universe.

\section{References}

[1] "Why the Moon is Getting Further Away from Earth." BBC News. Accessed 1 February, 2011. http://www.bbc.com/news/science-environment-12311119.

[2] Zhang San-hui 2008. University Physics - Mechanics, Thermodynamics. Beijing: Tsinghua University Press (in Chinese).

[3] Cui-xiang Zhong 2014. "Origin and Evolution of the
Moon.” In: Proceedings IAU Symposium No.298: 257.

[4] Cui-xiang Zhong 2016. "A Reason for the Earth Moving Away from the Sun and the Possibility to Escape Being Engulfed by a Red Giant Sun." American Journal of Astronomy and Astrophysics: 25-27.

[5] Chang, Kenneth, 13 March 2011. "Quake Moves Japan Closer to U.S. and Alters Earth's Spin.” The New York Times.

[6] Kostama, V. P., Kreslavsky, M. A., and Head, J. W. (June 3, 2006). "Recent High-Latitude Icy Mantle in the Northern Plains of Mars: Characteristics and Ages of Emplacement." Geophysical Research Letters 33 (11): L11201.

[7] Byrne, S., and Ingersoll, A. P. (2003). "A Sublimation Model for Martian South Polar Ice Features.” Science 299 (5609): 1051-1053. 